\title{
A Proposed Silicon Optical Electronic Integrated Circuit with Monolithic Integration of LED, OPFET and Receiver Circuit
}

\author{
Shiraz Afzal ${ }^{1}$, M. Zamin Ali Khan ${ }^{2}$ and Hussain Saleem ${ }^{3}$ \\ ${ }^{1}$ Electronic Engineering Department, Sir Syed University of Engineering \& Technology, Karachi, Pakistan \\ ${ }^{2}$ Main Communication Network Department, University of Karachi, Karachi, Pakistan \\ ${ }^{3}$ Department of Computer Science, University of Karachi, Karachi, Pakistan
}

\begin{abstract}
The paper presents an improved model of Silicon Optical Electronic Integrated Circuit (SOIC). The objective is to improve our OEIC model i.e. OEIC Circuit with Two Metal Layer Silicon Waveguide and Low Power Photonic Receiver Circuit by increasing responsitivity by increasing the quantum efficiency without changing the basic approach. This can be done by using optically controlled silicon MESFET also called OPFET. We also introduced the transparent indium tin oxide gate which when combines with MESFET, acts as a pre-amplifier in OEIC. The optically controlled silicon MESFET is integrated here having improved responsitivity, low gate to source capacitance, less signal delay, reduced noise and increased quantum efficiency.
\end{abstract}

Keywords: OEIC, OPFET, MESFET, SOIC, Quantum efficiency, Respositivity.

\section{INTRODUCTION}

In recent years, the considerable attention has been drawn by Optical Electronic IC (OEIC) development. Electrical coupling or join has proved to be a big problem for high speed data transmission. Therefore optical electronic Integrated Circuit is best choice that is introduced. This not only increases the bandwidth but also improves the speed and efficiency.

With the introduction of Optically Controlled Silicon MESFET (OPFETs), a very good responsitivity measure is achieved. The high speed operation of MESFET was first explained by [14] when it was compared with Avalanche Photo Diode (APD). The OPFET(s) show good performance on optical fiber as illustrated by [16]. Thus achieving low dispersion and very high speed. Further the transparent indium tin oxide indicates excellent device characteristic with high transconductance $g_{m}$ compares to metal gate MESFET [13] since the communication depends on the high bandwidth, low cost and distance. Therefore the "Figure of Merit" for communication FOM Communication is the product of bandwidth and distance as explained by [18] in Eq.(1).

FOM $_{\text {Communication }}=$ Bandwidth $\times$ Distance

But while considering $S i$, one can obtain the benefit by converging the electronics and photonics on silicon chip as [18] gives the "Figure of Merit" for silicon chip as the ratio of bandwidth to cost in Eq.(2) as:

FOM $_{\text {Silicon }}=\frac{\text { Bandwidth }}{\text { Cost }}$

Hence the dependency of FOM on the factors e.g. Bandwidth, Cost, Distance and Power are illustrated in Figure-1. Therefore in order to make the Silicon Optical Electronic Chip cost effective with low power consumption and high bandwidth, is proposed by introducing two metal layer silicon waveguide [1][15] as the silicon is readily available on earth and therefore the most feasible technology. In this paper the proposed OEIC consist of LED as light emitting device and the photodiode as the light detecting device, two metal layer silicon waveguide and MOSFET receiver circuit. This paper also present the silicon optical electronic integrated circuit which differs from the one as reported by [2] that instead of photodiode the OPFET is used. As a result a high speed with low dispersion is obtained [18]. 


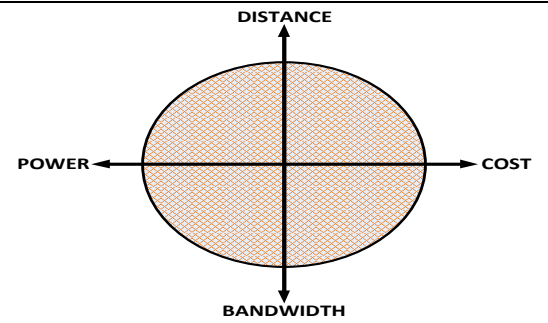

Figure-1: The dependency of FOM on the factors e.g. Bandwidth, Cost, Distance and Power

With the Introduction in Section-1, Secion-2 covers the discussion on Light Emitting Diode. Section-3 gives detail about Silicon OPFET. Section-4 explores the structure of Indium Tin Oxide Transparent Gate. Section-5 discusses the Silicon Waveguide, while Section-6 covers the schematic of Receiver Circuit. The Conclusion is presented in Section-7.

\section{LIGHT EMITTING DIODE}

With the development in the communication technology, substantial research work has been done in the silicon based light emitter like Nano-crystal, porous silicon, SiGe structure dislocation engineering, silicon dioxide super lattices, rare earth doping in silicon or silicon dioxide as explained by [17]. Reverse voltage(s) is applied for silicon light emitting diode to operate it in reverse mode for avalanche effect which operates above the breakdown voltage. The light emission of silicon LED was first reported in 1955 [3]. Lots of efforts were made in integrating the silicon $P N$ junction for the emission of visible light in standard VLSI (Very Large Scale Integration) technology [4][5][6]. The LED in this paper is fabricated with photodiode and waveguide using standard $0.35 \mu \mathrm{m}$ CMOS technology. The Light emitting diode is shown in the left side of Figure-2. It consists of a series of inter-digitated $p^{+}$octagonal rings and $n^{+}$octagonal rings con-centrically arranged around an $n^{+}$ center in the $n$-well. In order to reduce the breakdown voltage, the $n^{+}$octagonal ring is used to increase the light emitting area. The multiple $p^{+} / n$-well is used which act as a series of $P N$ junction. Further the $p^{+}$protective ring is provided in order to prevent the diffusive carrier.

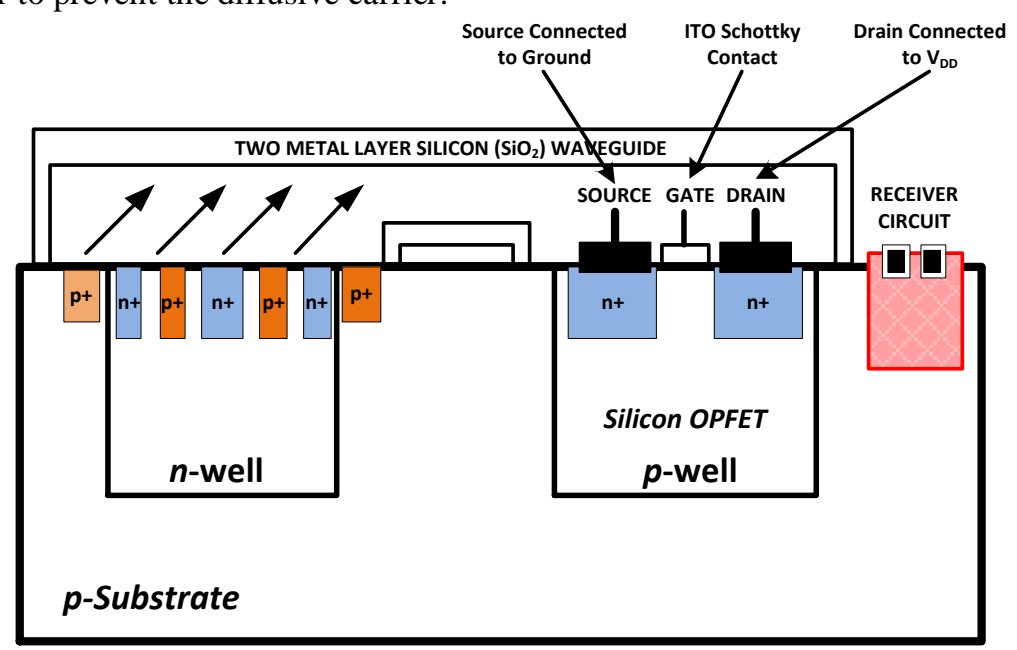

Figure-2: Proposed Model Layout of OEIC

\section{SILICON OPFET}

In the recent years, the silicon OPFET has drawn the considerable attention to emerge a promising light detector device for use in Optical Electronic Integrated Circuit. The right hand side of the Figure- 2 shows the optically controlled silicon MESFET which replaces the photodiode as it was previously reported by [2].

The theory of operation for Silicon controlled optical MESFET as reported by [11] while its analytical modeling has been described in [12] for details. The electron-hole pair is generated when the semiconductor device like OPFET is illuminated by photons with energy $E_{P h}=$ hv greater than band-gap of Silicon $1.12 \mathrm{eV}$ and smaller than the energy band-gap of Indium tin oxide i.e. $\sim 3.5 \mathrm{eV}$. Depending on the wavelength of the incident light and the structure of the device, several different photo-induced processes may occur. The primary photo effect along the Schottky junction (low injection case of incident photon flux) is photovoltaic. In this case, the photo-generated electron-hole pairs are separated by the Schottky junction built in electric field and move in different directions due to the space charge effect [13].

The electrons move towards the channel and holes move toward the surface. The holes recombine with traps or recombination centers, giving rise to a gate leakage current [16] [19]. Thus a photo voltage is developed 

across the junction, effectively reducing the depletion width in the channel. This reduction of the gate depletion region causes an increase in drain current for a given source-drain bias. Thus a photo voltage is developed across the junction, effectively reducing the depletion width in the channel. This reduction of the gate depletion causes an increase in drain current. Thus variation of depletion width directly affects the drain current. This optical modulation of drain current gives higher optical responsitivity in FET compared to a simple Schottky diode, which normally achieves an optical responsitivity of less than unity. Additional lesser photo-induced effects may be presented as well. For large gate resistances, a noticeable photo voltage is generated, supplementing the dark gate-source bias voltage. A similar photovoltaic effect can also occur at the channel substrate junction that can act as a photodiode but this effect can usually be ignored by assuming low injection conditions [13].

The Electric field swept out the photo-generated carrier in the depletion layer from gate to source and therefore contributes the photo current flow between drain and gate. The gate voltage changes due to the gate current which in-turn causes a change in the drain current [20]. The Gate made-up of transparent indium tin oxide (ITO) called TGFET (transparent gate MESFET) absorbs the optical radiation. Due to this effect electronhole pair are generated and due to drift the Carrier transport occur. The generated electron moves towards the $n$-channel as the gate biasing is applied. Whereas the hole move towards the surface. A reasonable channel thickness is used in order to absorb the maximum optical radiation as well as to prevent the loss of photogenerated carrier. The peak responsitivity and maximum output voltage of the $13 \mu \mathrm{m}$ gate OPFET were found to be at $620 \mathrm{~nm}$ and $1.0 \mathrm{MHz}$ [11]. Figure-3 depicts the graphical sketch of OPFET optical response i.e. Responsitivity versus optical wavelength in nanometer (nm).

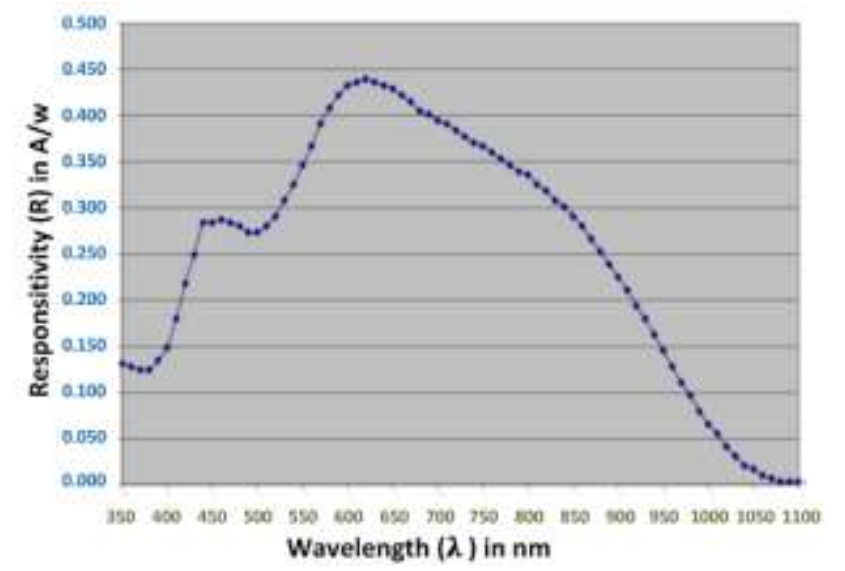

Figure-3: OPFET optical response; Responsitivity versus Optical wavelength

Another important factor for optically controlled silicon MESFET is the responsitivity which is given by Eq. (3) as:

$R=\frac{Q \cdot E \times \lambda \times q}{h c}$

Where $R$ is the respositivity in $\mathrm{A} / \mathrm{w}, Q . E$. is the quantum efficiency, $\lambda$ is the wavelength in $\mathrm{nm}, h$ is the Plank's constant and $q$ is the charge on electron. As the optical response reported by [11] the peak responsitivity is $0.439 \mathrm{~A} / \mathrm{w}$ occurs at $620 \mathrm{~nm}$, so the above equation will be given in Eq.(4):

$Q . E x=\frac{0.439 \mathrm{~A} / \mathrm{w} \times 1242 \mathrm{~nm} / \mathrm{A}}{620 \mathrm{~nm}}=88 \%$

So it shows that optically controlled silicon MESFET having high respositivity improves the quantum efficiency to $88 \%$ which is much higher than the older optoelectronic devices.

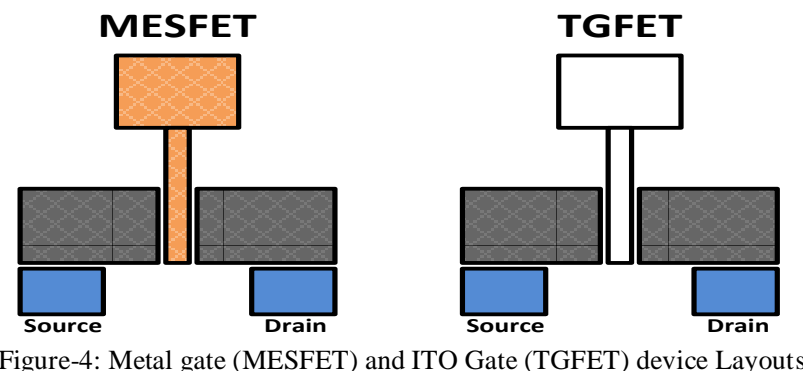

www.iosrjournals.org 


\section{INDIUM TIN OXIDE TRANSPARENT GATE}

In optoelectronic devices the optical coupling is an important factor for the detection of light. Here in our proposed OEIC, we have used Indium Tin Oxide transparent gate MESFET called TGFET instead of the conventional metal gate MESFET because TGFET contains the transparent area between the source and drain which make it more sensitive to optical radiation as compare to conventional metal gate.

This will provide greater optical efficiency due to the more active area available. As a result, the tremendous device characteristics are obtained [13]. Figure-4 depicts the phenomena of Metal gate and Indium Tin Oxide Gate layouts. The further detail is shown in Figure-2, where left hand side of our proposed model clearly shows the optical coupling with ITO for the detection of photon.

\section{SILICON WAVEGUIDE}

Rapid growth in the field of silicon photonics is made in the last few decades. A significant work has been done in integrating electronics and photonics on the same chip. Here in our proposed OEIC a two metal waveguide is implemented. The traditional ridge waveguide is not used here whose core refractive index is greater than cladding. High transmission efficiency could be achieved using traditional waveguide but it can't be fabricated by modern Ultra Large Scale Complementary Metal-Oxide-Semiconductor process (CMOS). Instead of using Silicon-on-Insulator (SOI) it uses silicon substrate widely as materials and it is essentially a planar process, which makes it difficult to form a step-shaped ridge waveguide. To solve this problem, here in our proposed structure, we have implemented the silicon dioxide waveguide that is compatible with standard CMOS process. It uses multiple layers of copper as interconnection and in between that the silicon dioxide are used acting as a dielectric insulating layer. The refractive index copper is smaller than silicon dioxide at $850 \mathrm{~nm}$ wavelength. So we can use copper as cladding and silicon dioxide as core to implement the waveguide [2].

\section{RECEIVER CIRCUIT}

A schematic diagram of the receiver circuit which is used to extract the photocurrent is shown in the Figure-5. The main component of this receiver circuit is the optically controlled MESFET (OPFET) which is connected in the common source configuration and act as a combination of both photo-detector and amplifier. As the MESFET is illuminated by the incident light, therefore the characteristics of MESFET are controlled by incident optical power. A comparator is used in the schematic that tells whether the voltage signal is high or low. Here the feedback resistance can be adjusted to vary the gain and obtain the maximum frequency response.

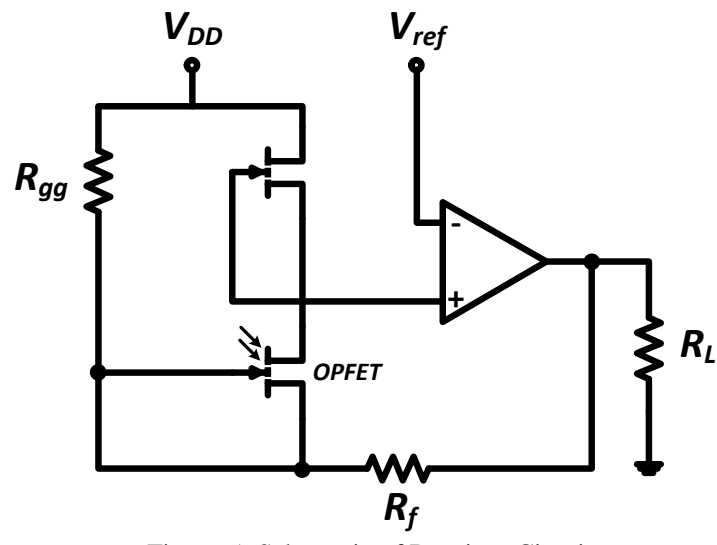

Figure-5: Schematic of Receiver Circuit

\section{CONCLUSION}

The proposed Optical Electronic Integrated Circuit (OEIC) is designed using the optically controlled silicon MESFET, two metal Layer Silicon waveguide and MESFET. The receiver circuit is also implemented with excellent quantum efficiency (88\%), less cost, improved performance due to high photocurrent, better respositivity and less noise. The research scope can be extended by introducing new material like Graphene or $\mathrm{GaN}$ would be extremely valuable for high frequency applications.

\section{REFERENCES}

[1] Beiju Haung \& et.al. "Monolithic Integration of Light Emiting Diodes, Photodetector and Receiver Circuit in Standard CMOS Technology", In Proc. IEEE 9th Intl. Conf. on Solid-State and Integrated-Circuit Technology, ICSICT, (2008).

[2] R. A Yotter, "Optimized CMOS Photodetector Structures for the Detection of Green Luminescent Probes", pp.43-49, Sensor and Actuator, B103, (2004).

[3] R. Newman, "Physics Review", (1955).

[4] L.W. Snyman, M. Du Plessis \& E. Seevinck, “IEEE Electron device Letter", (1999).

[5] M. Du Plessis, H.Aharoni, "Optical Materials", (2005). 
[6] H. Chen \& et.al., "A Silicon Light Emitting Devices in Standard CMOS Technology", IEEE 1st International Conference on Group IV Photonics, Hong Kong, (2004).

[7] A. Pauchard, P.A Besse, R.Popvic, “A Silicon Blue/UV Selective Stripe Shape”, Sensor and Actuators, A76, pp.172-177, (1999).

[8] Xuezhe Zheng, "Ultra-efficient $10 \mathrm{~GB} / \mathrm{s}$ Hybrid Integrated Silicon Photonic Transmitter and Receiver", (2011).

[9] D. Dobberpuhl, "The Design of A High Performance Low Power Microprocessor", Proc. of the IEEE Intl. Symp. on Low-Power Electronics and Design, (1996).

[10] M. Ingels, and M. S. J. Steyaert, “A 1-Gb/s, 0.7-um CMOS Optical Receiver with Full Rail-to-Rail Output Swing”, IEEE Journal Solid-State Circuits, 34(7), 971-977, (1999).

[11] S.N. Chattopadhyay \& et. al., "Optically Controlled Silicon MESFET Fabrication and Characterizations for Optical Modulator/Demodulator", Journal of Semiconductor Technology and Science, VOL.10, NO.3, pp.213-224, (2010).

[12] S. N. Chattopadhyay \& et. al., "Optically Controlled Silicon MESFET Modeling Considering Diffusion Process", Journal of Semiconductor Technology and Science, pp.196-208, (2007).

[13] A.H. Khalid and A.A. Rezazadeh, "Fabrication and Characterization of Transparent-Gate Field Effect Transistors using Indium Tin Oxide", IEEE Proceedings - Opto-electronics, Vol.143, No.1, pp.7-11, (1996).

[14] C. Baack et. al., "GaAs MESFET: A High-Speed Optical Detector", Electronics Letters, Vol.13, p.193, (1977).

[15] Shiraz Afzal, M. Zamin Ali Khan, Hussain Saleem, "A Proposed OEIC Circuit with Two Metal Layer Silicon Waveguide and Low Power Photonic Receiver Circuit”, IJCSI International Journal of Computer Science Issues, Vol. 9, Issue 5, No 1, September (2012).

[16] B.B. Pal and S.N. Chattopadhyay, "Time Dependent Analysis of An ION Implanted GaAs OPFET", IEEE Trans. Electron Devices, Vol. 42, p. 491, (1994).

[17] H. Liu \& et. al., "Silicon Light Emitting Device in CMOS Technology", Optoelectronic Letter, Vol.3, No.2. (2007).

[18] Kazumi Wada, Sungbong Park, and Yasuhiko Ishikawa, "Si Photonics and Fiber to the Home", Proceedings of the IEEE, Vol. 97,No. 7, July (2009).

[19] P. Chakrabarti, \& et. al., “An Improved Model of Ion-Implanted GaAs OPFET,” IEEE Trans. on Electron Devices, Vol. 39, No. 9, pp.2050-2059, (1992).

[20] T. Sugeta and Y. Mizushima, "High Speed Photoresponse Mechanism of A GaAs MESFET", Japanese Journal of Appl. Physics, Vol. 19, No. 1, pp. L27- L29, (1980).

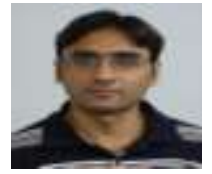

Shiraz Afzal is a full time faculty member at Sir Syed University of Engineering \& Technology, Karachi, Pakistan. He received his B.S. in Electronics from Sir Syed University of Engineering and Technology Karachi, Pakistan and M.E degree in Electronics with specialization in Micro-System design from NED University of Engineering and Technology Karachi, Pakistan in 2006 and 2012 respectively. His research interest includes Microelectronic circuit design. He is also a member of PEC.

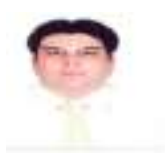

Dr. M. Zamin Ali Khan is a Head of Main Communication network department at University of Karachi. He has received B.E (Electrical Engineering) from NED University, Karachi, Pakistan and MS (Electrical and Computer Engineering) from Concordia University, Montreal, Canada and $\mathrm{PhD}$ in Computer Science from UoK. He has more than 18 years of experience of teaching and industry. He has worked in Victhom Human Bionics, Canada as an Engineer Scientist. Currently, he is a senior member of Pakistan Engineering Council, Canadian Engineering Council and IEEE. His research interest includes VLSI, Digital design, Digital signal processing and Analog front end of wireless devices.

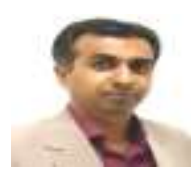

Hussain Saleem is currently Assistant Professor and Ph.D. Research Scholar at Department of Computer Science, University of Karachi, Pakistan. He received B.S. in Electronics Engineering from Sir Syed University of Engineering \& Technology, Karachi in 1997 and has done Masters in Computer Science from University of Karachi in 2001. He bears vast experience of about 15 years of University Teaching, Administration and Research in various dimensions of Computer Science. Hussain is the Author of several International Journal publications. His field of interest is Software Science, System Automation, Hardware design and engineering, and Simulation \& Modeling. He is senior member of Pakistan Engineering Council (PEC). 